\title{
Multiple MicroRNAs Ameliorate Hepatocyte Steatosis and Injury by Suppressing FABP1 Expression
}

\author{
Yun-li Wua,b Yi-Bing Zhu ${ }^{a} \quad$ Rong-dong Huang ${ }^{a} \quad$ Xian-e Peng ${ }^{b} \quad$ Xu Lin ${ }^{a, b}$ \\ ${ }^{a}$ Key Laboratory of Ministry of Education for Gastrointestinal Cancer, Fujian Medical University, Fuzhou, \\ ${ }^{\mathrm{b}}$ Fujian Key Laboratory of Tumor Microbiology, Fujian Medical University, Fuzhou, China
}

\section{Key Words}

Liver fatty acid-binding protein - MicroRNA • Steatosis • Nonalcoholic fatty liver disease

\begin{abstract}
Background/Aims: Liver fatty acid-binding protein (FABP1) is a key regulator of hepatic lipid metabolism. MicroRNAs (miRNAs) are thought to be involved in nonalcoholic fatty liver disease (NAFLD), and the underlying mechanism is largely unclear. We investigated whether miRNAs influence hepatocyte steatosis by regulating the FABPI gene. Methods: Candidate $F A B P 1$-targeting miRNAs were evaluated using luciferase reporter assay. FABP1 expression was measured using western blotting and quantitative reverse transcription-PCR. Intracellular lipid accumulation was measured based on Oil Red $O$ staining and intracellular triglyceride content. Hepatocyte injury was evaluated based on culture supernatant levels of alanine aminotransferase, aspartate aminotransferase, and intracellular adenosine triphosphate, and mitochondrial membrane potential. Results: Dicer1 knockdown significantly elevated FABP1 expression. In total, 68 miRNAs potentially targeting FABP1 were selected; of these, miR-3941, miR-4517, and miR-4672 directly targeted the FABP1 $3{ }^{\prime}$ untranslated region. Mimics of the three miRNAs substantially repressed $F A B P 1$ expression at translational level and led to HepG2 cell resistance to steatosis and cell injury induced by free fatty acids mixture, which rescue of FABP1 overexpression reversed. Conclusion: Our findings identify a novel mechanism by which miRNAs protect against hepatocyte steatosis and injury by downregulating FABP1 expression.

\section{Introduction}

Non-alcoholic fatty liver disease (NAFLD) is deemed the hepatic manifestation of metabolic syndrome, the characteristics of which include insulin resistance, dyslipidemia, hypertension, and type 2 diabetes $[1,2]$. The occurrence of NAFLD is mainly attributed to the accumulation of fat in the liver, and cirrhosis can follow; it is irreversible and may eventually 
develop into hepatocellular carcinoma $[3,4]$. However, many factors are involved in the complex process of liver steatosis; its pathogenesis is largely unknown. Recent cell studies and genetically modified mouse models have demonstrated that hepatic lipid metabolism and steatosis are crucially affected by peroxisome proliferator-activated receptor (PPAR)-c coactivator $1 \alpha$ and $1 \beta[5,6]$, receptor-interacting protein 140 [7], and liver fatty acid-binding protein [8] (FABP1 or L-FABP).

FABP1 belongs to a multi-gene FABP family of 14-15-kDa cytoplasmic proteins involved in the uptake, transport, and metabolism of cellular long-chain fatty acids and other lipid ligands [9]. The human FABP1 gene is localized in the p11.2 region of chromosome 2 [10]. FABP1 is abundant in the cytosol of liver parenchymal cells $[11,12]$ and represents approximately $0.2 \%$ of the total cytosolic proteins in the human hepatoblastoma cell line HepG2 [13]. Similar to its family members, FABP1 plays a central role in intracellular fatty acid transport and utilization [14] and is also involved in modulating mitosis [15], cell growth, and differentiation [16]. In vitro cell models and in vivo mouse models have indicated that FABP1 plays an important role in regulating hepatic lipid metabolism. HepG2 cell studies have indicated that $F A B P 1$ overexpression markedly increased the rate of fatty acid uptake. In contrast, FABP1 antisense RNA expression decreased fatty acid uptake significantly [17]. FABP1 $1^{-/}$mice fed a high-saturated fat, high-cholesterol "Western" diet were protected against diet-induced obesity and hepatic steatosis, reflecting an alteration in the kinetics of saturated fatty acid utilization $[18,19]$.

MicroRNAs (miRNAs) are single-stranded, 19-25-nucleotide long, non-coding RNAs that are involved in the regulation of numerous biological processes by base-pairing, usually imperfectly, to the 3' untranslated region (UTR) of a target mRNA, inhibiting it post-transcriptionally and occasionally leading to mRNA degradation [20, 21]. miRNAs can regulate the metabolism-related genes potentially involved in NAFLD pathogenesis. For example, the most highly expressed hepatic miRNA is miR-122, a regulator of cholesterol and fatty acid metabolism $[22,23,24]$; miR-10b regulates cellular steatosis levels through PPAR $\alpha$ [24]. Furthermore, mitochondrial dysfunction [25], oxidative stress [26], insulin resistance [27], and inflammation [28], generally considered critical components of NAFLD, are regulated by several miRNAs.

miRNAs regulating FABP1 expression have not been identified. Here, we determined whether miRNAs can regulate FABP1 gene expression and participate in FABP1-associated hepatic steatosis.

\section{Materials and Methods}

\section{Cell culture and clinical specimens}

HepG2 cells (HB-8065, ATCC, Manassas, VA) were maintained in Dulbecco's modified Eagle medium (Invitrogen, Carlsbad, CA) supplemented with $10 \%$ (v/v) fetal bovine serum (Invitrogen). Surgically resected adjacent normal liver tissues were obtained from 24 hepatocellular carcinoma (HCC) patients who underwent surgical resection. All samples were immediately frozen and stored in liquid nitrogen. The study protocol had all the appropriate approvals by the institutional review board and regulatory authorities. All patients had given informed and written consent.

\section{Bioinformatics analysis}

Possible FABP1-targeting miRNAs were predicted using four miRNA target-prediction tools: TargetScan, PicTar, miRanda, and RNA22. Bioinformatics analysis of the FABP1 3' UTR and all predicted miRNAs were considered potential targets for further analysis.

Construction of expression vectors

We constructed the pcDNA3.1-FABP1 vector by ligating the FABP1 cDNA into the HindIII and Xhol sites of pcDNA3.1-Hygro (+) (Invitrogen). FABP1 cDNA was amplified using reverse transcription-PCR from mRNA 


\section{Cellular Physiology Cell Physiol Biochem 2017;44:2243-2255 \begin{tabular}{l|l|l} 
and Biochemistry Published online: December 14, 2017 & $\begin{array}{l}\text { (c) } 2017 \text { The Author(s). Published by S. Karger AG, Basel } \\
\text { www.karger.com/cpb }\end{array}$ \\
\hline
\end{tabular}}

Wu et al.: Mirna Suppression of FABP1 Expression in Hepatocytes

isolated from HepG2 cells. The forward and reverse primers were 5'-CCCAAGCTTATGAGTTTCTCCGGCAAGTA3' and 5'-CCGCTCGAGTTAAATTCTCTTGCTGATTC-3', respectively.

\section{Luciferase reporter constructs and oligonucleotide transfection}

Wild-type and mutant miRNA-targeted FABP1 3' UTR were synthesized chemically by BoShang Biotech (Shanghai, China) and cloned downstream of a promoter-driven dual-luciferase reporter plasmid pmirGLO (Promega, Madison, WI). Table 2 shows the mutation sites.

The Dicer1 small interfering RNA (siRNA) mix and negative control (NC) siRNA were obtained from Santa Cruz Biotechnology (Santa Cruz, CA); Mimics and inhibitors of miR-3941, miR-4517, and miR-4672 were synthesized by RiboBio (Guangzhou, China). Oligonucleotide transfection was performed using X-tremeGENE siRNA Transfection Reagent (Roche Diagnostics, GmbH, Mannheim, Germany).

\section{miRNA screening and luciferase assay}

For the miRNA screening, HepG2 cells were seeded in 12-well plates. After 24-h incubation, the cells were co-transfected with $1 \mu \mathrm{g}$ pmirGLO reporter plasmid carrying wild-type or mutant FABP1 3' UTR and $100 \mathrm{pmol} N \mathrm{NC}$ or mimic. The luciferase activity was measured $48 \mathrm{~h}$ after transfection using the dual-luciferase reporter assay (Promega) according to the manufacturer's recommendations. Luminescence was measured using a luminometer (Orion II Microplate Luminometer, Berthold Detection Systems, Pforzheim, Germany). All assays were performed in triplicate at minimum.

RNA extraction and quantitative reverse transcription (RT)-PCR ( $q P C R$ )

Total RNA was extracted using TRIzol (Invitrogen), and reverse-transcribed using the ExScript RTPCR Kit (TaKaRa, Shiga, Japan) according to manufacturer's instruction. qPCR was performed with the ABI StepOne Real-Time PCR System (Applied Biosystems, Foster City, CA) and the SYBR Premix Ex Taq Kit (TaKaRa) in accordance with the manufacturer's instructions. Glyceraldehyde-3-phosphate dehydrogenase $(G A P D H)$ served as the internal control. Each sample was analyzed in triplicate. The relative FABP1 mRNA level was calculated by normalization to the endogenous GAPDH mRNA expression prior to comparative analysis using the comparative threshold cycle $\left(2^{-\Delta \Delta C t}\right)$ method. The respective forward and reverse primers used were 5'-CAAGTTCACCATCACCGCTG-3' and 5'-ATTATGTCGCCGTTGAGTTCG-3' (FABP1), and 5'-TGCACCACCAACTGCTTAGC-3' and 5'-AGCTCAGGGATGACCTTGCC-3' (GAPDH).

Total miRNA form frozen tissues was extracted using the miRNeasy Mini Kit (Qiagen, Hilden, German) and cDNA was synthesized using miScript Reverse Transcription Kit (Qiagen). Expression levels of miR3941, miR-4517, and miR-4672 were quantified using a miRNA-specifc miScript Primer Assay (Qiagen) in combination with the miScript SYBR Green PCR Kit (Qiagen). SNORD6 (U6 snRNA) was used as an internal control.

\section{Western blotting}

Protein $(30 \mu \mathrm{g})$ was subjected to $12 \%$ sodium dodecyl sulfate-polyacrylamide gel electrophoresis, and electrophoretically transferred to a polyvinylidene fluoride membrane (Millipore, Billerica, MA). Protein blots were incubated separately with antibodies against Dicer1 (No. 3363, 1:500, Cell Signaling Technology, Beverly, MA), FABP1 (HPA028275, 1:100, Sigma-Aldrich, St. Louis, M0), or $\beta$-actin (1:4000, Sigma-Aldrich), probed with the appropriate alkaline phosphatase-conjugated secondary antibody, and detected using chemiluminescence. Immunoreactive protein bands were visualized by adding CDP-Star reagents (Roche Diagnostics, $\mathrm{GmbH}$ ). Band signal intensities were quantified using Quantity One densitometric software (Bio-Rad, Hercules, CA).

\section{Cellular steatosis}

Sodium palmitate (catalog\# P9767) and sodium oleate (catalog\# 07501) were obtained from SigmaAldrich. Fat-overloading induction of HepG2 cells was performed mainly according to previously established methods [29], where cells at $80 \%$ confluence were exposed to a mixture of unsaturated (oleate) and saturated (palmitate) long-chain free fatty acids (FFA) at a ratio of 2:1. Fatty acid-free bovine serum albumin (BSA) was used as the control. To assess the influence of miRNAs on cellular steatosis, the cells were treated with FFA mixture after 48-h transfection with miRNA mimics or inhibitors. Following incubation with the FFA mixture, cellular lipid accumulation was measured based on Oil Red O staining and triglyceride (TG) levels. 


\section{Cellular Physiology Cell Physiol Biochem 2017;44:2243-2255 \\ \begin{tabular}{l|l|l} 
and Biochemistry Published ondine: December 14, 2017 & $\begin{array}{l}\text { C } 2017 \text { The Author(s). Published by S. Karger AG, Basel } \\
\text { www.karger.com/cpb }\end{array}$
\end{tabular}}

Wu et al.: Mirna Suppression of FABP1 Expression in Hepatocytes

Oil Red $O$ staining

Oil Red 0 staining was performed using standard procedures as previously described [30]. Briefly, HepG2 cells were plated onto glass microscope slides, fixed with 5\% paraformaldehyde for 15 minutes, and stained with Oil Red $\mathrm{O}$ and hematoxylin.

\section{Biochemical analysis}

The levels of cell supernatant alanine aminotransferase (ALT) and aspartate aminotransferase (AST) were determined using a commercial kit (Cat.\# 1045-717 and 1055-717, respectively, Shanghai Kehua Bio-Engineering, Shanghai, China) using a HITACHI 7020 automated chemical analyzer (Tokyo, Japan) according to the manufacturer's instructions. Intracellular TG content was determined using a commercial kit (TR0100, Sigma-Aldrich) according to the manufacturer's instructions, and normalized to the protein content by bicinchoninic acid assay (Pierce, Rockford, IL).

\section{Assessment of mitochondrial membrane potential $(\Delta \psi m)$}

Changes in $\Delta \psi \mathrm{m}$ were assessed under a fluorescence microscope using the mitochondria-specific dual-fluorescence probe JC-1 (10 $\mu \mathrm{g} / \mathrm{ml}$, Invitrogen) according to manufacturer's instructions. JC- 1 is a ratiometric dye that is internalized as a monomer (green fluorescence, excitation/emission $=485 / 530 \mathrm{~nm}$ ) and is concentrated by respiring mitochondria with negative inner membrane potential into J-aggregates (red fluorescence, excitation/emission $=535 / 590 \mathrm{~nm}$ ). Consequently, mitochondrial depolarization (i.e., loss of $\Delta \psi \mathrm{m}$ ) is indicated by a decreased red/green fluorescence ratio.

Measurement of cellular adenosine triphosphate (ATP) levels

Intracellular ATP levels were determined using a bioluminescent somatic cell ATP assay kit (SigmaAldrich) according to the manufacturer's instructions. Briefly, cells were mixed with somatic cell ATPreleasing reagent and ATP assay mix. The amount of luminescence emitted from each reaction was measured immediately with a luminometer (Orion II Microplate Luminometer, Berthold Detection Systems); ATP levels in each sample were calculated from ATP standard curves. Data are expressed as nmol ATP per mg protein.

\section{Statistical analysis}

The differences between two groups were analyzed using a Student $t$-test. All values are expressed as the mean \pm standard deviation (SD) of triplicate experiments. $P<0.05$ was considered statistically significant.

\section{Results}

miRNAs downregulated FABP1 gene expression

To investigate whether miRNAs regulate FABP1, we knocked down Dicer1, a key regulator of miRNA processing (Fig. 1A, B). Dicer1 downregulation significantly elevated the levels of both mRNA (Fig. 1C) and protein (Fig. 1D) FABP1, indicating that miRNAs can regulate $F A B P 1$ expression.

To investigate whether miRNAs directly modulate $F A B P 1$, we introduced a luciferase reporter with the wild-type FABP1 3' UTR (pmirGLO-FABP13'UTR) into HepG2 cells and found that $F A B P 1$ expression levels were substantially increased (Fig. 1E), indicating that pmirGLO-FABP13'UTR might bind to the FABP1-targeting miRNAs, freeing the FABP1 mRNA from endogenous miRNA repression and possibly increasing FABP1 protein expression. These observations suggest that miRNAs modulate FABP1 expression by directly targeting its 3' UTR.

Screening for miRNAs that directly target the FABP1 3' UTR

The TargetScan, RNA22, PicTar, and miRanda miRNA target-prediction tools were used to predict candidate $F A B P 1$-targeting miRNAs. A pool of 68 miRNAs that potentially target FABP1 was identified (Table 1). HepG2 cells were co-transfected with pmirGLO-FABP13'UTR together with the candidate miRNA mimics, and luciferase activity was measured $48 \mathrm{~h}$ after transfection. Seven of the 68 miRNAs, i.e., miR-16, miR-134, miR-490-3p, miR-767-5p, miR3941, miR-4517, and miR-4672, downregulated the luciferase activity significantly as com- 
pared with the empty vector control (Fig. 2A, B). Fig. 2C depicts the target sequence location of the seven miRNAs in the FABP1 3' UTR. We subsequently performed serial mutations of these potential sites (Table 2), and constructed mutant FABP1 3' UTR luciferase reporter plasmids. The luciferase reporter assay showed that miR-3941, miR-4517, and miR-4672 binding site mutations abrogated the suppressor effects. However, miR-16, miR-767-5p, miR-134, and miR-490-3P showed no rescue effect on luciferase activity (Fig. 2D). Taken together, the results suggest that miR-3941, miR-4517, and miR-4672 can modulate $F A B P 1$ by directly targeting the corresponding $3^{\prime}$ UTR sites.

miR-3941, miR-4517, and miR-4672 inhibited FABP1 expression

To determine whether miR-3941, miR-4517, and miR4672 can regulate endogenous

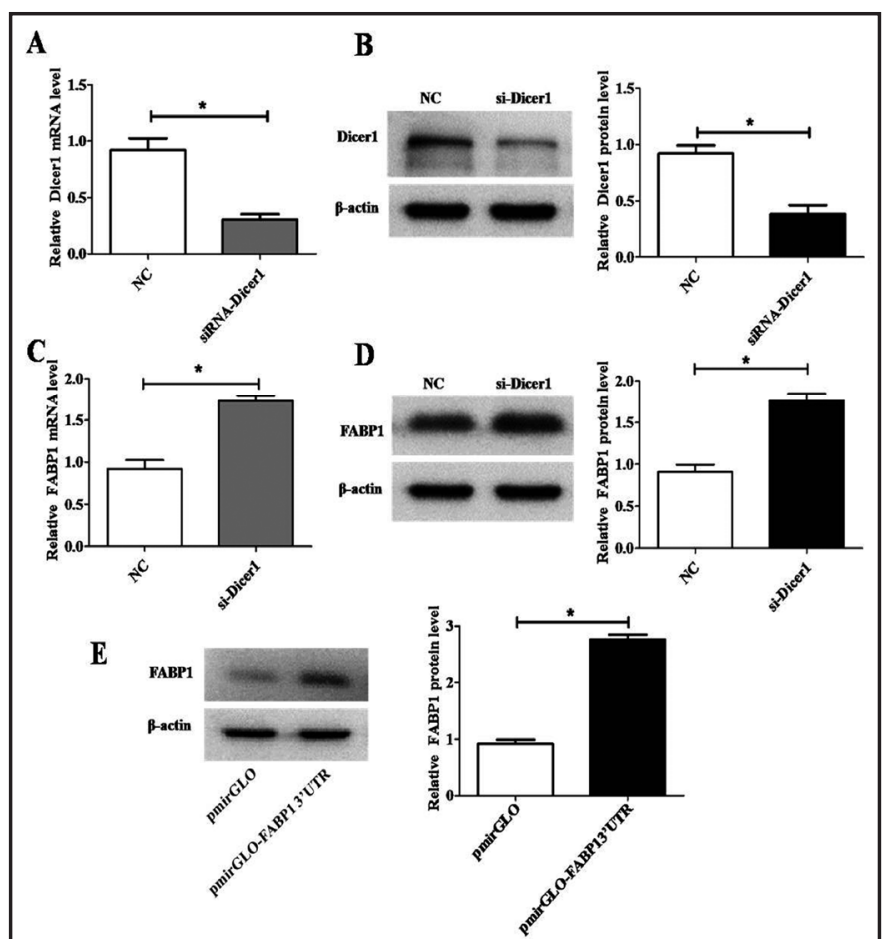

Fig. 1. FABP1 is regulated by miRNAs. $(A, B)$ Dicer1 mRNA (A) and protein (B) levels in HepG2 cells after siRNA (si-Dicer) knockdown. FABP1 mRNA (C) and protein (D) levels in HepG2 cells transfected with si-Dicer or NC. (E) Western blot analysis of FABP1 protein levels after pmirGLO-FABP13'UTR transfection in HepG2 cells. $\beta$-Actin was the loading control for the western blotting; GAPDH was the internal control for the qPCR. ${ }^{*} \mathrm{P}<0.05$ versus control. FABP1, we treated HepG2

cells with their miRNA mimics or inhibitors and evaluated the FABP1 mRNA and protein levels using qPCR and western blotting, respectively. The miRNA mimics downregulated FABP1 protein levels substantially as compared to the NC (Fig. 3A). In contrast, silencing the miRNAs significantly increased FABP1 expression (Fig. 3B). Interestingly, the miRNA mimics and inhibitors did not alter FABP1 mRNA levels significantly compared to the NC (Fig. 3C), indicating that the three miRNAs predominantly function at translational level. We also examine the clinical relevance of the miR-3941, miR-4517, miR-4672 and FABP1 in human liver tissues. As shown in Fig. 3E-F, specimens expressing low-level FABP1 (1\# 12\#, 12 cases), exhibited high miR-3941 (66.7\%, 8 cases), miR-4517 (58.3\%, 7 cases) and miR4672 (75\%, 9 cases) expression, whereas samples with high FABP1 expression (13\# 24\#, 12 cases) showed low-level miR-3941 (58.3\%, 7 cases), miR-4517 (75\%, 9 cases) and miR4672 (83.3\%, 10 cases), respectively (each $\mathrm{P}<0.05$ ). The results suggest that miR-3941, miR-4517, miR-4672 and FABP1 expression levels were negatively correlated in HepG2 cell line and liver tissues.

miR-3941, miR-4517, and miR-4672 alleviated FFA-induced hepatocyte steatosis

To investigate the influence of miR-3941, miR-4517, and miR-4672 on FFA-induced hepatocyte steatosis, we treated HepG2 cells with $0.5 \mathrm{mM}$ FFA mixture (oleate/palmitate [2:1]) to induce in vitro cellular steatosis. Oil Red 0 staining (Fig. 4A) revealed that FFA, a functional FABP1 inducer [12,31], induced substantial lipid accumulation in the cells, and cells transfected with miR-3941, miR-4517, or miR-4672 mimic had significantly lower intracellular lipid levels compared to the control. In contrast, rescue of FABP1 overexpression in cells treated with miR-3941, miR-4517, or miR-4672 mimic (confirmed by western 
Table 1. Possible FABP1-3'UTR-targeting miRNAs predicted by four target-prediction tools

\begin{tabular}{|c|c|c|}
\hline miRNA & miRBase ID & Sequence \\
\hline miR-203 & MIMAT0031890 & AGUGGUUCUUAACAGUUCAACAGUU \\
\hline miR-323-3p & MIMAT0000755 & CACAUUACACGGUCGACCUCU \\
\hline miR-329 & MIMAT0001629 & AACACACCUGGUUAACCUCUUU \\
\hline miR-362-3p & MIMAT0004683 & AACACACCUAUUCAAGGAUUCA \\
\hline miR-466 & MIMAT0015002 & AUACACAUACACGCAACACACAU \\
\hline miR-495 & MIMAT0022924 & GAAGUUGCCCAUGUUAUUUUCG \\
\hline $\operatorname{miR}-499 a-5 p$ & MIMAT0002870 & UUAAGACUUGCAGUGAUGUUU \\
\hline miR-556-3p & MIMAT0004793 & AUAUUACCAUUAGCUCAUCUUU \\
\hline miR-590-3p & MIMAT0004801 & UAAUUUUAUGUAUAAGCUAGU \\
\hline miR-603 & MIMAT0003271 & CACACACUGCAAUUACUUUUGC \\
\hline miR-3166 & MIMAT0015040 & CGCAGACAAUGCCUACUGGCCUA \\
\hline miR-3941 & MIMAT0018357 & UUACACACAACUGAGGAUCAUA \\
\hline $\operatorname{miR}-4517$ & MIMAT0019054 & AAAUAUGAUGAAACUCACAGCUGAG \\
\hline miR-4672 & MIMAT0019754 & UUACACAGCUGGACAGAGGCA \\
\hline miR-4771 & MIMAT0019925 & AGCAGACUUGACCUACAAUUA \\
\hline miR-4775 & MIMAT0019931 & UUAAUUUUUUGUUUCGGUCACU \\
\hline $\operatorname{miR}-29 \mathrm{~b}$ & MIMAT0004514 & GCUGGUUUCAUAUGGUGGUUUAGA \\
\hline $\operatorname{miR}-29 c$ & MIMAT0004673 & UGACCGAUUUCUCCUGGUGUUC \\
\hline miR-98 & MIMAT0000096 & UGAGGUAGUAAGUUGUAUUGUU \\
\hline miR-103 & MIMAT0007402 & UCAUAGCCCUGUACAAUGCUGCU \\
\hline miR-107 & MIMAT0000104 & AGCAGCAUUGUACAGGGCUAUCA \\
\hline miR-134 & MIMAT0000447 & UGUGACUGGUUGACCAGAGGGG \\
\hline miR-182 & MIMAT0000259 & UUUGGCAAUGGUAGAACUCACACU \\
\hline miR-195 & MIMAT0000461 & UAGCAGCACAGAAAUAUUGGC \\
\hline miR-340 & MIMAT0000750 & UCCGUCUCAGUUACUUUAUAGC \\
\hline miR-509-5p & MIMAT0004779 & UACUGCAGACAGUGGCAAUCA \\
\hline miR-512-3p & MIMAT0002823 & AAGUGCUGUCAUAGCUGAGGUC \\
\hline miR-520a-3p & MIMAT0002834 & AAAGUGCUUCCCUUUGGACUGU \\
\hline miR-612 & MIMAT0003280 & GCUGGGCAGGGCUUCUGAGCUCCUU \\
\hline miR-661 & MIMAT0003324 & UGCCUGGGUCUCUGGCCUGCGCGU \\
\hline miR-16 & MIMAT0000069 & UAGCAGCACGUAAAUAUUGGCG \\
\hline miR-28-5p & MIMAT0000085 & AAGGAGCUCACAGUCUAUUGAG \\
\hline miR-30e & MIMAT0000692 & UGUAAACAUCCUUGACUGGAAG \\
\hline miR-125a-5p & MIMAT0000443 & UCCCUGAGACCCUUUAACCUGUGA \\
\hline miR-143 & MIMAT0000435 & UGAGAUGAAGCACUGUAGCUC \\
\hline miR-186 & MIMAT0000456 & CAAAGAAUUCUCCUUUUGGGCU \\
\hline $\operatorname{miR}-208 \mathrm{~b}$ & MIMAT0004960 & AUAAGACGAACAAAAGGUUUGU \\
\hline miR-337-3p & MIMAT0000754 & CUCCUAUAUGAUGCCUUUCUUC \\
\hline miR-369-3p & MIMAT0000721 & AAUAAUACAUGGUUGAUCUUU \\
\hline miR-372 & MIMAT0026484 & CCUCAAAUGUGGAGCACUAUUCU \\
\hline miR-422a & MIMAT0001339 & ACUGGACUUAGGGUCAGAAGGC \\
\hline miR-490-3p & MIMAT0002806 & CAACCUGGAGGACUCCAUGCUG \\
\hline miR-513-3p & MIMAT0004777 & UAAAUUUCACCUUUCUGAGAAGG \\
\hline miR-520b & MIMAT0002843 & AAAGUGCUUCCUUUUAGAGGG \\
\hline miR-520d-3p & MIMAT0002856 & AAAGUGCUUCUCUUUGGUGGGU \\
\hline miR-520e & MIMAT0002825 & AAAGUGCUUCCUUUUUGAGGG \\
\hline miR-520f & MIMAT0002830 & AAGUGCUUCCUUUUAGAGGGUU \\
\hline miR-595 & MIMAT0003263 & GAAGUGUGCCGUGGUGUGUCU \\
\hline miR-609 & MIMAT0003277 & AGGGUGUUUCUCUCAUCUCU \\
\hline miR-613 & MIMAT0003281 & AGGAAUGUUCCUUCUUUGCC \\
\hline miR-629 & MIMAT0003298 & GUUCUCCCAACGUAAGCCCAGC \\
\hline miR-647 & MIMAT0003317 & GUGGCUGCACUCACUUCCUUC \\
\hline miR-767-5p & MIMAT0003882 & UGCACCAUGGUUGUCUGAGCAUG \\
\hline $\operatorname{miR}-22$ & MIMAT0000077 & AAGCUGCCAGUUGAAGAACUGU \\
\hline miR-30a & MIMAT0000087 & UGUAAACAUCCUCGACUGGAAG \\
\hline miR-125b & MIMAT0000423 & UCCCUGAGACCCUAACUUGUGA \\
\hline $\operatorname{miR}-140-3 p$ & MIMAT0004597 & UACCACAGGGUAGAACCACGG \\
\hline miR-200a & MIMAT0000682 & UAACACUGUCUGGUAACGAUGU \\
\hline miR-217 & MIMAT0000274 & UACUGCAUCAGGAACUGAUUGGA \\
\hline miR-302d & MIMAT0000718 & UAAGUGCUUCCAUGUUUGAGUGU \\
\hline miR-342-5p & MIMAT0004694 & AGGGGUGCUAUCUGUGAUUGA \\
\hline miR-345 & MIMAT0000772 & GCUGACUCCUAGUCCAGGGCUC \\
\hline miR-373 & MIMAT0000725 & ACUCAAAAUGGGGGCGCUUUCC \\
\hline miR-378 & MIMAT0000731 & CUCCUGACUCCAGGUCCUGUGU \\
\hline miR-425 & MIMAT0003393 & AAUGACACGAUCACUCCCGUUGA \\
\hline $\operatorname{miR}-449 \mathrm{~b}$ & MIMAT0003327 & AGGCAGUGUAUUGUUAGCUGGC \\
\hline miR-454 & MIMAT0003884 & ACCCUAUCAAUAUUGUCUCUGC \\
\hline miR-486-5p & MIMAT0002177 & UCCUGUACUGAGCUGCCCCGAG \\
\hline
\end{tabular}

Table 2. Conserved binding sequence of top seven miRNAs in FABP1-3'UTR. Mutant bases are underlined

\begin{tabular}{|c|c|c|}
\hline No. & Binding sequence & Site \\
\hline 1 & 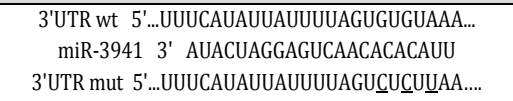 & $27-34$ \\
\hline 2 & 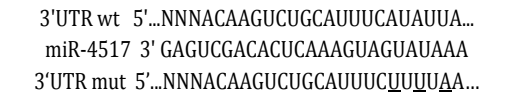 & $14-21$ \\
\hline 3 & 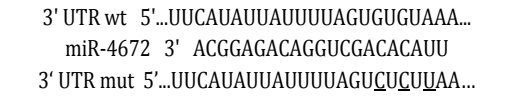 & $28-34$ \\
\hline 4 & $\begin{array}{c}\text { 3' UTR wt 5'...NNNACAAGUCTGCAUUUCAUAUUAUUUU.... } \\
\text { miR-16 3' GCGGUUAUAAAUGCACGACGAU } \\
\text { 3' UTR mut 5'....NNNACAAGUGACGAUUUCATAUUAUUUU... }\end{array}$ & 7-10 \\
\hline 5 & $\begin{array}{c}\text { 3' UTR wt 5' NNNACAAGUCUGCAUUUCAUUAUUAUUUUA... } \\
\text { miR-767-5p 3' GUACGAGUCUGUUGGUACCACGU } \\
\text { 3' UTRmut 5' NNNACAUGGUCACGUUUUCAUAUUAUUUUA... }\end{array}$ & 4-11 \\
\hline 6 & $\begin{array}{l}\text { 3'UTR wt } 5{ }^{\prime} \text { ACAAGUCUGCAUUUCAUAUUAUUUUUA... } \\
\text { miR-134 3' GGGGAGACCAGUUGGUCAGUGU } \\
3 \text { UTR mut } \quad 5 \text { ' ACAAGUCUGCAUUAGUUUUUAUUUUA... }\end{array}$ & $14-18$ \\
\hline 7 & $\begin{array}{c}\text { 3' UTR wt } \quad 5^{\prime} \text {...AAUAAAAGUGAACUUUGUUUUA.... } \\
\text { miR-490-3p 3'-GUCGUACCUCAGGAGGUCCAAC } \\
\text { 3' UTR mut } \quad 5^{\prime} \text {...AAUAAAGUGAAGUUUCAAUUA... }\end{array}$ & $55-61$ \\
\hline
\end{tabular}

blotting, Fig. 4B) markedly restored lipid accumulation. We obtained similar results for intracellular TG content, where rescue of FABP1 overexpression reversed the inhibitory effects of the miRNA mimics (Fig. 4C). The results indicate that miR3941, miR-4517, and miR-4672 alleviate FFA-induced hepatocyte steatosis by downregulating FABP1 expression.

miR-3941, miR-4517, and miR-4672 expression ameliorated FFA-induced hepatocyte injury

Lipid accumulation in hepatocytes leads to mitochondrial dysfunction, which is regarded as a critical component of NAFLD [25, 32, 33]. we examined HepG2 cell $\Delta \psi \mathrm{m}$ and ATP levels to investigate the effects of miRNAs on mitochondrial function. The control cells had brightly stained mitochondria that emitted red fluorescence (Fig. 5A). FFA-treated cells had diffuse green fluorescence in the cytoplasm, indicating dissipation of the $\Delta \psi \mathrm{m}$. miRNAtreated cells had substantially increased red fluorescence, indicating recovery of $\Delta \psi \mathrm{m}$ 


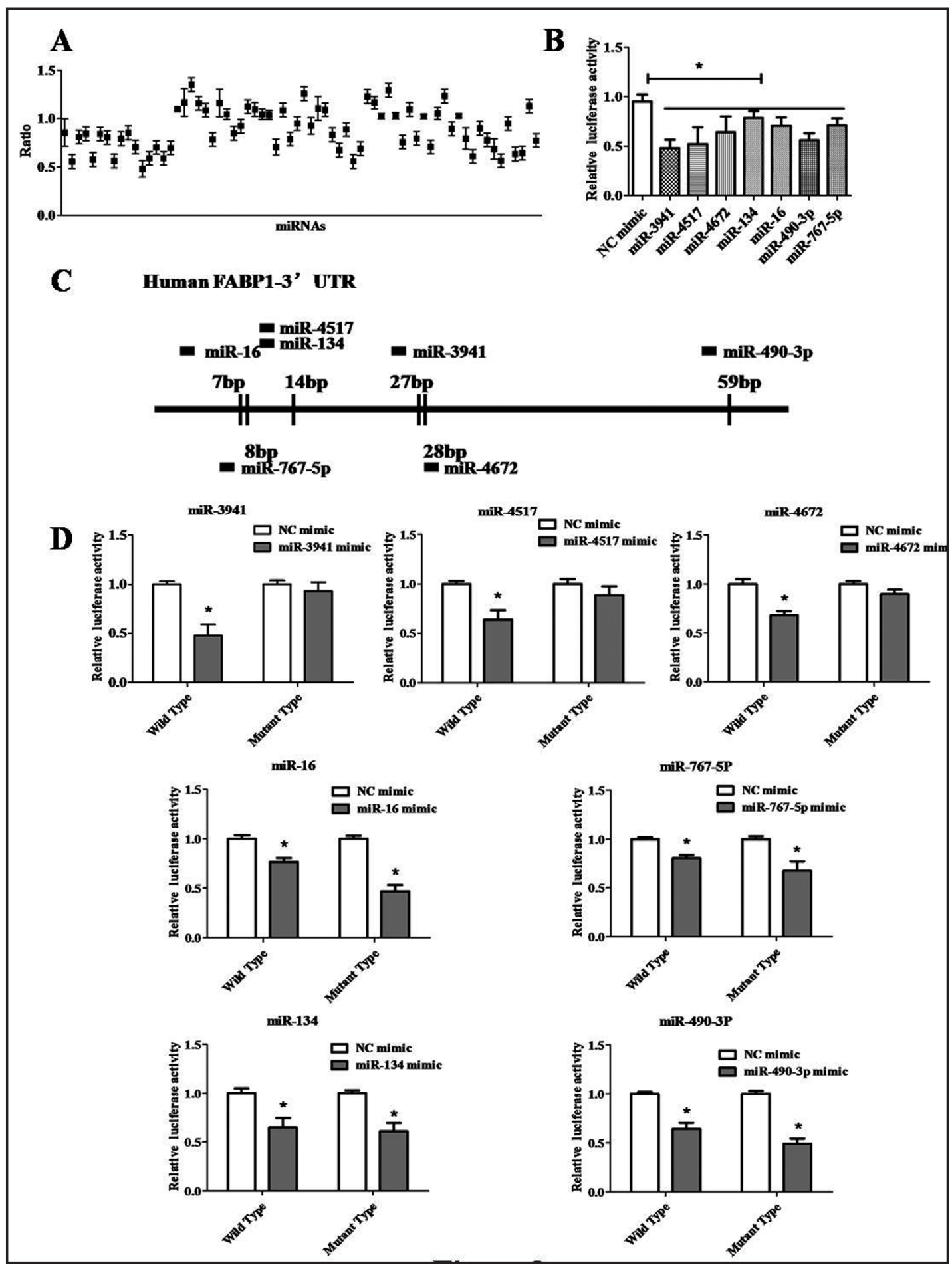

Fig. 2. Screen for candidate miRNAs targeting the FABP1 3' UTR. (A, B) Luciferase assay results. Of 68 miRNAs, seven significantly decreased pmirGLO-FABP13'UTR luciferase activity compared with the NC. (C) Schematic diagram of the binding sites of the FABP1-regulating miRNAs in the wild-type FABP1 3' UTR. (D) Luciferase activity in HepG2 cells co-transfected with wild-type or mutant FABP1 3' UTR luciferase reporter plasmids ( $1 \mu \mathrm{g} /$ well) and siRNA (100 pmol) or NC siRNA (100 pmol). The luciferase activity of each sample was normalized to Renilla luciferase activity. The normalized luciferase activity of the transfected NC was set as relative luciferase activity of 1 . Data are the mean \pm SD of three independent experiments. ${ }^{*} \mathrm{P}<0.05$ versus control. 


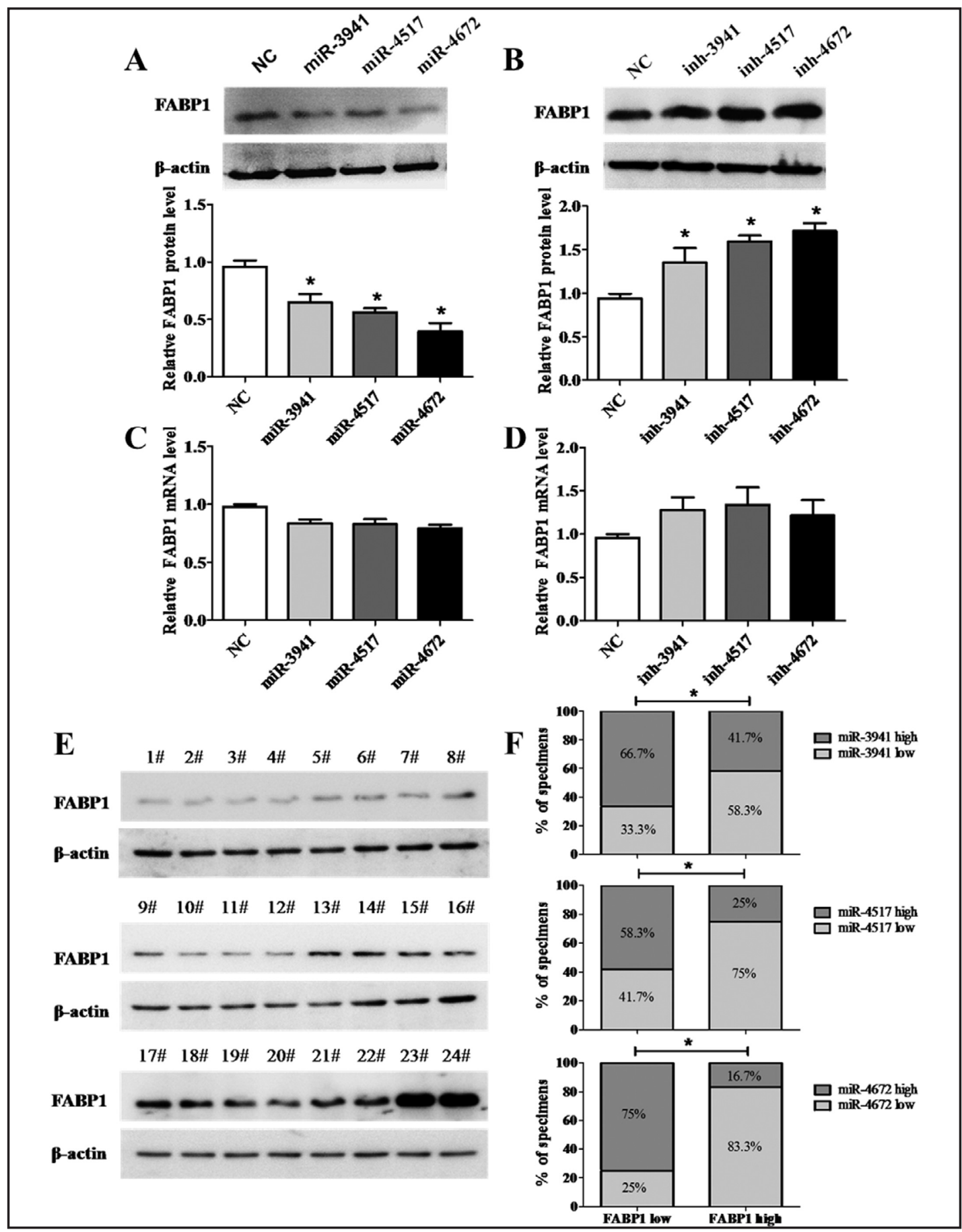

Fig. 3. FABP1-targeting miRNAs can inhibit FABP1 protein expression in HepG2 cells and liver tissues. (A, B) Western blot analysis of FABP1 protein levels in HepG2 cells transfected with miR-3941, miR-4517, or miR-4672 mimics (A) or their inhibitors (B). $\beta$-Actin was the internal control. Data are the mean \pm SD of three independent experiments. (C, D) qPCR analysis of FABP1 mRNA levels in HepG2 cells transfected with miR-3941, miR-4517, or miR-4672 mimics (C) or their inhibitors (D). Expression was normalized against the endogenous control GAPDH. ${ }^{*} \mathrm{P}<0.05$. (E, F) Association of miRNAs with FABP1 expression level in liver tissues. FABP1 protein level was analysed by western blot, $\beta$-actin was the internal control (E). Percentage of specimens showing low or high miRNAs level in relation to the expression levels of FABP1 (F). miR-3941, miR-4517, or miR-4672 level was analysed by qPCR in 24 liver tissues. Expression levels were normalized by U6 snRNA level. ${ }^{*} \mathrm{P}<0.05$.

\section{KARGER}


Fig. 4. $\mathrm{miR}-3941$, miR-4517, and miR-4672 increase lipid accumulation in HepG2 cells by regulating FABP1. (A) Oil Red 0 staining showing the different responses of HepG2 cells transfected with miR-3941, miR-4517, and miR-4672 mimics and/or overexpressing FABP1 ( p c D N A 3.1 FABP1) to FFA-induced cellular lipid accumulation. Representative images are shown. Red staining indicates neutral lipids. Original magnification, $\times 100$. (B) Western blot analysis of FABP1 protein levels. The ratio of FABP1 protein levels in treated cells relative to that in cells transfected with control pcDNA3.1Hygro(+) was calculated densitometrically after normalization to the level of $\beta$-actin. Values are

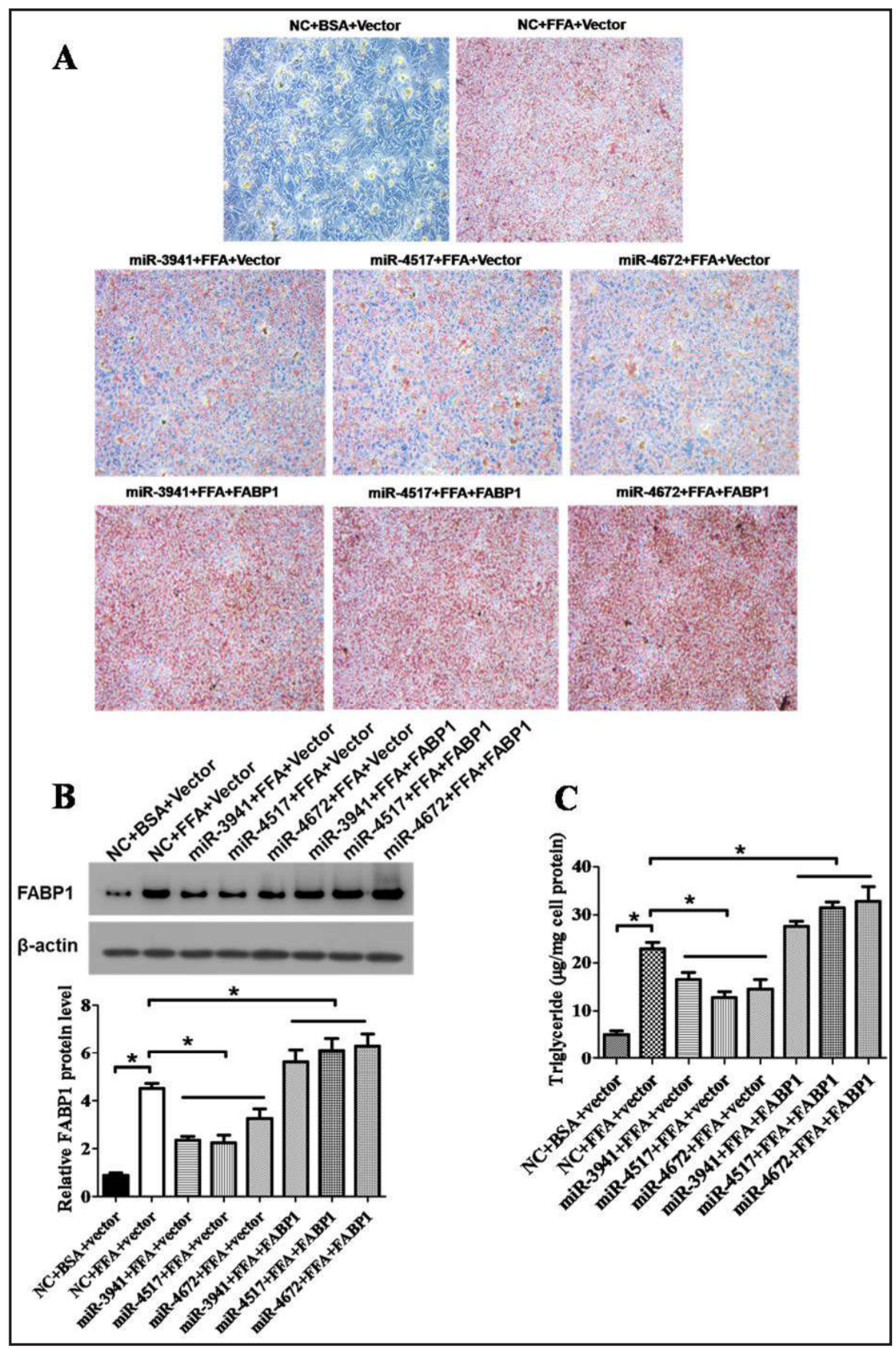
mean $\pm \mathrm{SD}, \mathrm{n}=3$.

(C) Measurement of TG content in HepG2 cell lysates. TG levels were normalized to protein concentration $(\mu \mathrm{g} / \mathrm{mg})$ and are reported as the mean \pm SD of three separate experiments. ${ }^{*} \mathrm{P}<0.05$.

loss. In contrast, rescue of $F A B P 1$ overexpression significantly increased $\triangle \psi \mathrm{m}$ loss in the miRNA-treated cells. Similar results were obtained for the intracellular ATP levels, which represent mitochondrial function damage and cell injury. The ATP levels in FFA-treated cells were markedly decreased (Fig. 5B), whereas miRNA mimics significantly increased ATP levels, and rescue of FABP1 overexpression reversed the inhibitory effects on ATP levels.

Mitochondrial dysfunction results in hepatocyte injury, for which AST and ALT are regarded as markers. FFA significantly increased AST levels (Fig. 5C), but had no significant

\section{KARGER}


Fig. 5. Effects of miRNA-mediated FABP1 repression on FFAinduced hepatocyte injury. (A) $\Delta \psi \mathrm{m}$ in HepG2 cells was assessed using JC-1 dye under fluorescence microscopy. Red fluorescence (Jaggregates) indicates functional mitochondria; green fluorescence (JC-1 monomers) indicates dysfunctional mitochondria. Original magnification, $\times 200$. (B) Bioluminescent somatic cell assay measurement of intracellular ATP levels (nmol ATP/mg protein) in HepG2 cells. (C, D) AST (C) and ALT (D) levels in HepG2 cell supernatant. Values are the mean \pm SD of three separate experiments performed in duplicate. ${ }^{*} \mathrm{P}<0.05$ versus control.

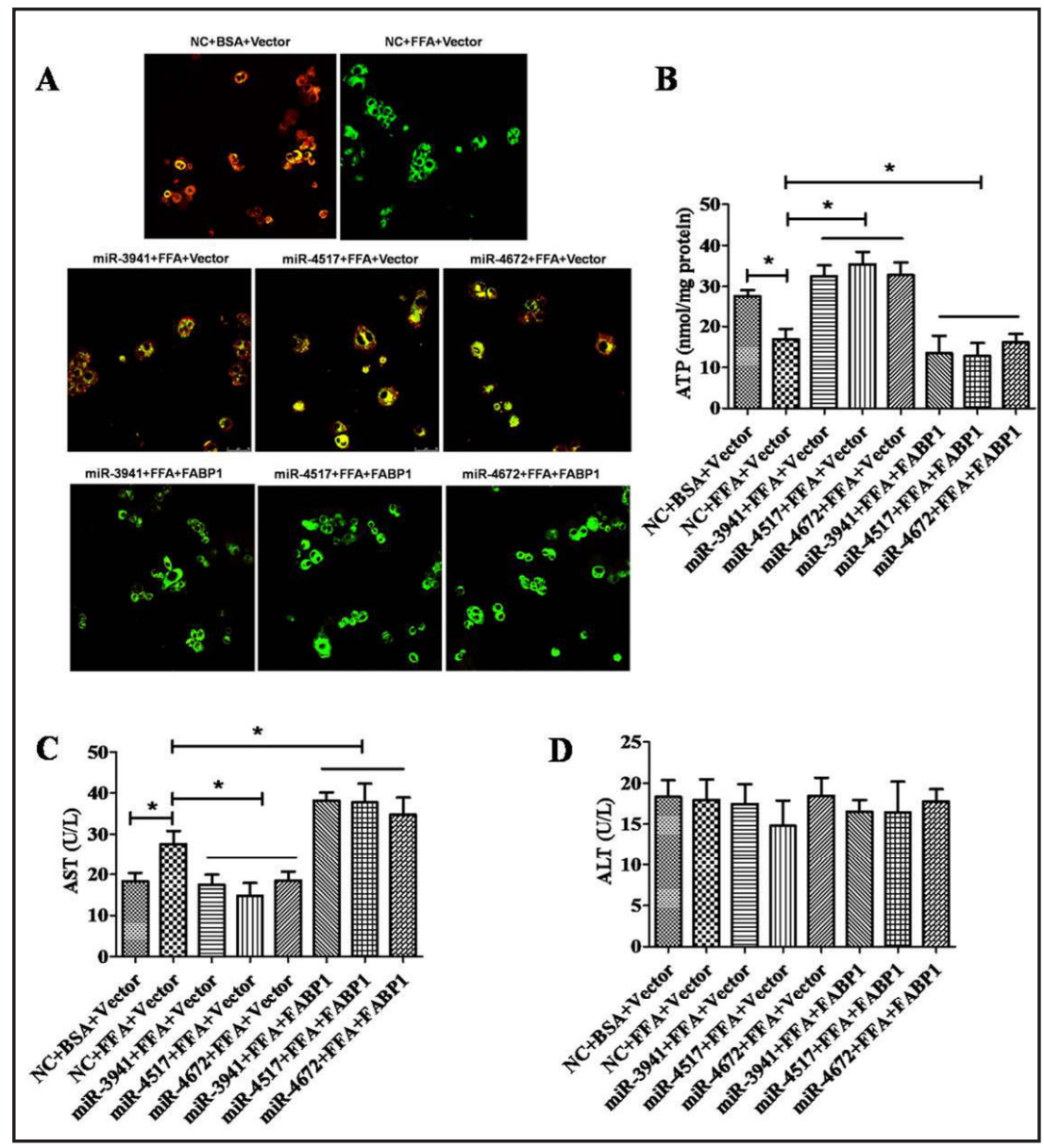

effects on ALT levels (Fig. 5D), which is consistent with the report by Ding et al [34].. FFAtreated cells transfected with miR-3941, miR-4517, and miR-4672 mimics had significantly decreased AST levels (Fig. 5C). As expected, rescue of FABP1 overexpression in cells treated with miR-3941, miR-4517, and miR-4672 mimics yielded opposite results (Fig. 5C). These findings suggest that miR-3941, miR-4517, and miR-4672 alleviate FFA-induced mitochondrial dysfunction and hepatocyte injury by downregulating FABP1 expression.

\section{Discussion}

NAFLD has attracted considerable attention due to its high morbidity. It is characterized by excessive TG accumulation in hepatocytes [35]. Prolonged NAFLD is associated with steatohepatitis, which can result in end-stage liver disease [36, 37]. Increasing evidence indicates that miRNAs are associated with hepatic steatosis by altering lipid metabolism [38, 39]. In vitro cell models and in vivo mouse models have shown that FABP1 plays an important role in regulating hepatic lipid metabolism $[17,18,19]$. Accordingly, we hypothesized that miRNAs may be involved in FABP1-induced hepatic steatosis.

To address this hypothesis, we first knocked down the expression of Dicer1, a key regulator of miRNA processing. Both FABP1 mRNA and protein levels were significantly elevated, indicating that miRNAs regulate FABP1. miRNAs negatively regulate their target mRNAs post-transcriptionally by binding to the $3^{\prime}$ UTR. Further, we found that FABP1 expression levels were substantially increased following transfection with a luciferase 


\section{Cellular Physiology Cell Physiol Biochem 2017;44:2243-2255

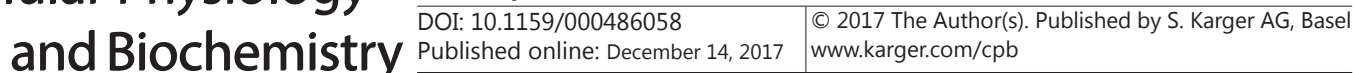 \\ Wu et al.: Mirna Suppression of FABP1 Expression in Hepatocytes}

reporter carrying the wild-type FABP1 3' UTR. Therefore, we conclude that miRNAs modulate FABP1 expression by directly targeting its 3' UTR. We used TargetScan, RNA22, PicTar, and miRanda to identify the miRNAs associated with FABP1 expression regulation, which predicted 68 human miRNAs that might target $F A B P 1$. The specific binding effect between the predicted miRNAs and the FABP1 3' UTR was confirmed by co-transfection of HepG2 cells with a luciferase reporter plasmid containing wild-type or mutant FABP1 3' UTR and the candidate miRNAs. Only miR-3941, miR-4517, and miR-4672 directly targeted the FABP1 3' UTR, which suggests that stringent site conservation is important in miRNAmediated FABP1 regulation. In addition, the miRNAs suppressed FABP1 protein levels, but not FABP1 mRNA levels, which indicates that the three miRNAs regulate FABP1 expression at translational level. FABP1 plays an important role in NAFLD pathogenesis by governing hepatocyte lipid metabolism. Studies on HepG2 cells and rat hepatoma cells have indicated that FABP1 markedly increases the rate of fatty acid uptake [17, 40]. Moreover, the Thr94Ala mutation in the FABP1 gene, which should abolish fatty acid binding, decreases TG levels as compared to that in wild-type cells when incubated with extracellular fatty acids [41]. In a transgenic mouse model, ablation of the Fabp1 gene impaired the ability of the liver to efficiently import and transfer fatty acids to glycerolipid biosynthesis, resulting in reduced hepatic TG accumulation [42]. These changes were not due to the inability to upregulate fatty acid oxidation or TG synthesis, but rather resulted from decreased rates of hepatic fatty acid uptake and trafficking in the face of short-term (48-h) mobilization of adipose TG stores and increased fatty acid availability [42]. Germline FABP1 $1^{-/-}$mice exhibited decreased hepatic TG content with altered fatty acid uptake kinetics [42]. Interestingly, FABP1/- mice had altered saturated fatty acid utilization kinetics, and were protected against "Western" diet-induced obesity and hepatic steatosis $[18,19]$. Proteomic screens have revealed that FABP1 is overexpressed in obese subjects with simple steatosis, along with paradoxically decreased expression in the progressive versus mild forms of nonalcoholic steatohepatitis [19]. In the present functional studies, we use an in vitro model of cellular steatosis to elucidate the important role of miR-3941, miR-4672, and miR-4517 in regulating hepatocyte lipid metabolism, where the results confirm that miR-3941, miR-4672, and miR-4517 mimics dramatically reduce FFA-induced lipid accumulation and intracellular TG levels by downregulating FABP1 expression in HepG2 cells. Moreover, it has been well-documented that fat accumulation in hepatocytes can induce liver damage $[43,44]$; our study also shows that miR-3941, miR-4517, and miR-4672 alleviate FFA-induced mitochondrial dysfunction and hepatocyte injury by downregulating FABP1 expression.

In summary, we demonstrate that miR-3941, miR-4672, and miR-4517 ameliorate FFA-induced hepatocyte steatosis and injury by suppressing FABP1 expression. Our study provides new understanding of a molecular mechanism underlying hepatocyte steatosis from the miRNA perspective and suggests that miR-3941, miR-4672, and miR-4517 could be of promising therapeutic value for NAFLD.

\section{Acknowledgements}

This work was supported by the grants from Foundation for Distinguished Young Scholars of Fujian Provincial University (Grant number 2015B025) and National Natural Science Foundation of China (Grant number 81271822).

\section{Disclosure Statement}

All authors declare that they have no Disclosure Statements. 


\section{Cellular Physiology Cell Physiol Biochem 2017;44:2243-2255 \begin{tabular}{l|l|l} 
and Biochemistry Published online: December 14, 2017 & $\begin{array}{l}\text { (c) } 2017 \text { The Author(s). Published by S. Karger AG, Basel } \\
\text { www.karger.com/cpb }\end{array}$ \\
\hline
\end{tabular}}

Wu et al.: Mirna Suppression of FABP1 Expression in Hepatocytes

\section{References}

1 Angulo P: Nonalcoholic fatty liver disease. N Engl J Med 2002;346:1221-1231.

2 de Alwis NM, Day CP: Non-alcoholic fatty liver disease: the mist gradually clears. J Hepatol 2008;48 Suppl 1:S104-112.

-3 Starley BQ Calcagno CJ, Harrison SA: Nonalcoholic fatty liver disease and hepatocellular carcinoma: a weighty connection. Hepatology 2010;51:1820-1832.

4 Anstee QM, Targher G, Day CP: Progression of NAFLD to diabetes mellitus, cardiovascular disease or cirrhosis. Nat Rev Gastroenterol Hepatol 2013;10:330-344.

5 Leone TC, Lehman JJ, Finck BN, Schaeffer PJ, Wende AR, Boudina S, Courtois M, Wozniak DF, Sambandam N, Bernal-Mizrachi C, Chen Z, Holloszy JO, Medeiros DM, Schmidt RE, Saffitz JE, Abel ED, Semenkovich CF, Kelly DP: PGC-1alpha deficiency causes multi-system energy metabolic derangements: muscle dysfunction, abnormal weight control and hepatic steatosis. PLoS Biol 2005;3:e101.

-6 Sonoda J, Mehl IR, Chong LW, Nofsinger RR, Evans RM: PGC-1beta controls mitochondrial metabolism to modulate circadian activity, adaptive thermogenesis, and hepatic steatosis. Proc Natl Acad Sci U S A 2007;104:5223-5228.

7 Leonardsson G, Steel JH, Christian M, Pocock V, Milligan S, Bell J, So PW, Medina-Gomez G, Vidal-Puig A, White R, Parker MG, O’Malley BW: Nuclear receptor corepressor RIP140 regulates fat accumulation. Proc Natl Acad Sci U S A 2004;101:8437-8442.

-8 Wu YL, Peng XE, Zhu YB, Yan XL, Chen WN, Lin X: Hepatitis B Virus X Protein Induces Hepatic Steatosis by Enhancing the Expression of Liver Fatty Acid Binding Protein. J Virol 2015;90:1729-1740.

-9 Hertzel AV, Bernlohr DA: The mammalian fatty acid-binding protein multigene family: molecular and genetic insights into function. Trends Endocrinol Metab 2000;11:175-180.

-10 Zimmerman AW, Veerkamp JH: New insights into the structure and function of fatty acid-binding proteins. Cell Mol Life Sci 2002;59:1096-1116.

$>11$ Storch J, Corsico B: The emerging functions and mechanisms of mammalian fatty acid-binding proteins. Annu Rev Nutr 2008;28:73-95.

12 Wolfrum C, Ellinghaus P, Fobker M, Seedorf U, Assmann G, Borchers T, Spener F: Phytanic acid is ligand and transcriptional activator of murine liver fatty acid binding protein. J Lipid Res 1999;40:708-714.

13 Gibbons GF: A comparison of in-vitro models to study hepatic lipid and lipoprotein metabolism. Curr Opin Lipidol 1994;5:191-199.

14 Schroeder F, Jolly CA, Cho TH, Frolov A: Fatty acid binding protein isoforms: structure and function. Chem Phys Lipids 1998;92:1-25.

15 Sorof S: Modulation of mitogenesis by liver fatty acid binding protein. Cancer Metastasis Rev 1994;13:317336.

16 Schroeder F, Atshaves BP, Starodub O, Boedeker AL, Smith RR, 3rd, Roths JB, Foxworth WB, Kier AB: Expression of liver fatty acid binding protein alters growth and differentiation of embryonic stem cells. Mol Cell Biochem 2001;219:127-138.

$>17$ Wolfrum C, Buhlmann C, Rolf B, Borchers T, Spener F: Variation of liver-type fatty acid binding protein content in the human hepatoma cell line HepG2 by peroxisome proliferators and antisense RNA affects the rate of fatty acid uptake. Biochim Biophys Acta 1999;1437:194-201.

-18 Newberry EP, Xie Y, Kennedy SM, Luo J, Davidson NO: Protection against Western diet-induced obesity and hepatic steatosis in liver fatty acid-binding protein knockout mice. Hepatology 2006;44:1191-1205.

19 Newberry EP, Kennedy SM, Xie Y, Luo J, Davidson NO: Diet-induced alterations in intestinal and extrahepatic lipid metabolism in liver fatty acid binding protein knockout mice. Mol Cell Biochem 2009;326:79-86.

20 Ambros V: The functions of animal microRNAs. Nature 2004;431:350-355.

21 Lewis BP, Shih IH, Jones-Rhoades MW, Bartel DP, Burge CB: Prediction of mammalian microRNA targets. Cell 2003;115:787-798.

22 Esau C, Davis S, Murray SF, Yu XX, Pandey SK, Pear M, Watts L, Booten SL, Graham M, McKay R, Subramaniam A, Propp S, Lollo BA, Freier S, Bennett CF, Bhanot S, Monia BP: miR-122 regulation of lipid metabolism revealed by in vivo antisense targeting. Cell Metab 2006;3:87-98.

-23 Cheung O, Puri P, Eicken C, Contos MJ, Mirshahi F, Maher JW, Kellum JM, Min H, Luketic VA, Sanyal AJ: Nonalcoholic steatohepatitis is associated with altered hepatic MicroRNA expression. Hepatology 2008;48:1810-1820. 


\section{Cellular Physiology Cell Physiol Biochem 2017;44:2243-2255 \begin{tabular}{l|l|l} 
and Biochemistry Published online: December 14, 2017 & $\begin{array}{l}\text { (c) } 2017 \text { The Author(s). Published by S. Karger AG, Basel } \\
\text { www.karger.com/cpb }\end{array}$ \\
\hline
\end{tabular}}

Wu et al.: Mirna Suppression of FABP1 Expression in Hepatocytes

24 Zheng L, Lv GC, Sheng J, Yang YD: Effect of miRNA-10b in regulating cellular steatosis level by targeting PPAR-alpha expression, a novel mechanism for the pathogenesis of NAFLD. J Gastroenterol Hepatol 2010;25:156-163.

25 Li P, Jiao J, Gao G, Prabhakar BS: Control of mitochondrial activity by miRNAs. J Cell Biochem 2012;113:1104-1110.

26 Tang H, Lee M, Sharpe O, Salamone L, Noonan EJ, Hoang CD, Levine S, Robinson WH, Shrager JB: Oxidative stress-responsive microRNA-320 regulates glycolysis in diverse biological systems. FASEB J 2012;26:47104721.

27 Williams MD, Mitchell GM: MicroRNAs in insulin resistance and obesity. Exp Diabetes Res 2012;2012:484696.

-28 Roy S, Sen CK: MiRNA in innate immune responses: novel players in wound inflammation. Physiol Genomics 2011;43:557-565.

-29 Gomez-Lechon MJ, Donato MT, Martinez-Romero A, Jimenez N, Castell JV, O'Connor JE: A human hepatocellular in vitro model to investigate steatosis. Chem Biol Interact 2007;165:106-116.

-30 Joshi-Barve S, Barve SS, Amancherla K, Gobejishvili L, Hill D, Cave M, Hote P, McClain CJ: Palmitic acid induces production of proinflammatory cytokine interleukin-8 from hepatocytes. Hepatology 2007;46:823830.

31 Newberry EP, Davidson NO: Liver Fatty Acid Binding Protein (L-FABP) as a Target for the Prevention of High Fat Diet Induced Obesity and Hepatic Steatosis. Endocrinol Metab Agents Med Chem 2009;9:30-37.

-32 Pessayre D, Fromenty B: NASH: a mitochondrial disease. J Hepatol 2005;42:928-940.

-33 Caldwell SH, Swerdlow RH, Khan EM, Iezzoni JC, Hespenheide EE, Parks JK, Parker WD Jr: Mitochondrial abnormalities in non-alcoholic steatohepatitis. J Hepatol 1999;31:430-434.

-34 Ding J, Li M, Wan X, Jin X, Chen S, Yu C, Li Y: Effect of miR-34a in regulating steatosis by targeting PPARalpha expression in nonalcoholic fatty liver disease. Sci Rep 2015;5:13729.

-35 Adams LA, Lindor KD: Nonalcoholic fatty liver disease. Ann Epidemiol 2007;17:863-869.

-36 Powell EE, Jonsson JR, Clouston AD: Steatosis: co-factor in other liver diseases. Hepatology 2005;42:5-13.

37 Browning JD, Horton JD: Molecular mediators of hepatic steatosis and liver injury. J Clin Invest 2004;114:147-152.

-38 Ortega FJ, Mercader JM, Catalan V, Moreno-Navarrete JM, Pueyo N, Sabater M, Gomez-Ambrosi J, Anglada R, Fernandez-Formoso JA, Ricart W, Frühbeck G, Fernández-Real JM: Targeting the circulating microRNA signature of obesity. Clin Chem 2013;59:781-792.

-39 Arner E, Mejhert N, Kulyte A, Balwierz PJ, Pachkov M, Cormont M, Lorente-Cebrian S, Ehrlund A, Laurencikiene J, Heden P, Dahlman-Wright K, Tanti JF, Hayashizaki Y, Rydén M, Dahlman I, van Nimwegen E, Daub CO, Arner P: Adipose tissue microRNAs as regulators of CCL2 production in human obesity. Diabetes 2012;61:1986-1993.

40 Linden D, Lindberg K, Oscarsson J, Claesson C, Asp L, Li L, Gustafsson M, Boren J, Olofsson SO: Influence of peroxisome proliferator-activated receptor alpha agonists on the intracellular turnover and secretion of apolipoprotein (Apo) B-100 and ApoB-48. The Journal of biological chemistry 2002;277:23044-23053.

41 Gao N, Qu X, Yan J, Huang Q Yuan HY, Ouyang DS: L-FABP T94A decreased fatty acid uptake and altered hepatic triglyceride and cholesterol accumulation in Chang liver cells stably transfected with L-FABP. Mol Cell Biochem 2010;345:207-214.

-42 Newberry EP, Xie Y, Kennedy S, Han X, Buhman KK, Luo J, Gross RW, Davidson NO: Decreased hepatic triglyceride accumulation and altered fatty acid uptake in mice with deletion of the liver fatty acid-binding protein gene. J Biol Chem 2003;278:51664-51672.

43 Bugianesi E, Leone N, Vanni E, Marchesini G, Brunello F, Carucci P, Musso A, De Paolis P, Capussotti L, Salizzoni M, Rizzetto M: Expanding the natural history of nonalcoholic steatohepatitis: from cryptogenic cirrhosis to hepatocellular carcinoma. Gastroenterology 2002;123:134-140.

44 Yeh MM, Brunt EM: Pathological features of fatty liver disease. Gastroenterology 2014;147:754-764. 